\title{
Effect of Dietary Clostridium butyricum Supplementation on Growth Performance, Intestinal Barrier Function, Immune Function, and Microbiota Diversity of Pekin Ducks
}

\author{
Yanhan Liu ${ }^{1,2}$, Cun Liu ${ }^{2}$, Keying An ${ }^{1}$, Xiaowei Gong ${ }^{1}$ and Zhaofei Xia ${ }^{1, *}$ \\ 1 College of Veterinary Medicine, China Agricultural University, Beijing 100193, China; vetlyh@163.com (Y.L.); \\ s20193050785@cau.edu.cn (K.A.); gxw@cau.edu.cn (X.G.) \\ 2 Shandong Provincial Center for Animal Disease Control, Ji'nan 250100, China; liucun89@163.com \\ * Correspondence: zhaofeixiacau@163.com; Tel.: +86-10-62733781
}

check for updates

Citation: Liu, Y.; Liu, C.; An, K.; Gong, X.; Xia, Z. Effect of Dietary Clostridium butyricum Supplementation on Growth Performance, Intestinal Barrier Function, Immune Function, and Microbiota Diversity of Pekin Ducks. Animals 2021, 11, 2514. https:// doi.org/10.3390/ani11092514

Academic Editors:

Gabriel De La Fuente,

Joaquim Balcells

and Ahmad Reza Seradj

Received: 18 July 2021

Accepted: 21 August 2021

Published: 26 August 2021

Publisher's Note: MDPI stays neutral with regard to jurisdictional claims in published maps and institutional affiliations.

Copyright: (c) 2021 by the authors. Licensee MDPI, Basel, Switzerland. This article is an open access article distributed under the terms and conditions of the Creative Commons Attribution (CC BY) license (https:// creativecommons.org/licenses/by/ $4.0 /)$.
Simple Summary: In poultry farming, the use of prophylactic antibiotics can lead to increased resistance, so probiotics are a good alternative. Clostridium butyricum (C. butyricum) has been widely used to improve the gut health of animals. Therefore, we carried out the current study of Pekin ducks supplemented with C. butyricum for a period of 42 days. Here, we found a clear increase in the growth performance of Pekin ducks supplemented with C. butyricum. Moreover, a high level of secretory IgA, IgM, IgG, IL-4, and IL-10 and comparatively higher short-chain fatty acids (SCFAs) and intestinal tight junction changes were found in Pekin ducks supplemented with C. butyricum. The gut microbial diversity of Pekin ducks supplemented with C. butyricum was clearly different than that of Pekin ducks fed a non-C. butyricum diet. In conclusion, our findings suggest that $400 \mathrm{mg} / \mathrm{kg}$ C. butyricum supplementation improved the intestinal health of Pekin ducks by increasing the $\alpha$ diversity of intestinal microbiota, enhancing the SCFAs contents, and strengthening the intestinal barrier function and immune systems indicating that $400 \mathrm{mg} / \mathrm{kg}$ C. butyricum might be a preferable antibiotic alternative for commercial application.

\begin{abstract}
Clostridium butyricum (C. butyricum) is increasingly being used to test the promotion of the gut health of animals. However, the modes of action for such applications for waterfowl remain unclear. Thus, we investigated whether or not intestinal barrier function, immune-related gene expression, and the diversity of the intestinal microbiota in Pekin ducks varied under C. butyricum supplementation. A total of 500 ducks were randomly assigned into five treatments supplemented with basal diets containing: either 0 (group Control), 200 (group CB200), 400 (group CB400) and 600 (group CB600) mg/kg C. butyricum or $150 \mathrm{mg} / \mathrm{kg}$ aureomycin (group A150) for 42 days. In comparison with the control group, C. butyricum supplementation enhanced the growth performance and intestinal villus height of Pekin ducks at $42 \mathrm{~d}$. Serum immune indexes and fecal short-chain fatty acids (SCFAs) were all improved at both $21 \mathrm{~d}$ and $42 \mathrm{~d}$ after C. butyricum addition. The $m R N A$ expression levels of Mucin2, Zonula occludens-1 (ZO-1), Caudin-3, and Occludin increased at $21 \mathrm{~d}$ and $42 \mathrm{~d}$ and the mRNA expression levels of IL-4 and IL-10 only increased at $42 \mathrm{~d}$ after C. butyricum addition. Dietary C. butyricum also resulted in an increase in the number of diversities of operational taxonomic units (OTUs), and an increase in the $\alpha$-diversity of intestinal microbiota. The addition of $C$. butyricum altered the composition of the intestinal microbiota from $21 \mathrm{~d}$ to $42 \mathrm{~d}$. The relative abundance of Firmicutes and Bacteroidetes showed little changes among groups; however, the relative abundance of Firmicutes / Bacteroidetes were found to have been significantly different between the $21 \mathrm{~d}$ and $42 \mathrm{~d}$. C. butyricum administration improved the intestinal health of Pekin ducks by increasing the diversity of intestinal microbiota, enhancing the SCFAs contents, and strengthening the intestinal barrier function and immune systems. The optimal dietary supplementation dosage was recommended as $400 \mathrm{mg} / \mathrm{kg}$ in the diet.
\end{abstract}

Keywords: C. butyricum; intestine microbial; Pekin duck; SCFAs; tight junction 


\section{Introduction}

Probiotics have been found to be able to improve digestive function and prevent leaky intestines or intestinal inflammation. Clostridium butyricum (C. butyricum) is considered a Gram-positive obligate anaerobic probiotic which can produce butyric acid and form endospores, and has been documented as normal intestinal flora of healthy animals [1]. C. butyricum can tolerate high bile, high temperature environments, and acidic environments of the digestive tract, thus, it can be used as an effective feed additive [2]. C. butyricum can produce bacteriocin, lipoteichoic acid, hydrogen, and these prebiotics can help to improve intestinal anti-oxidation and anti-bacterial functions in animals [3]. C. butyricum is able to increase growth performance, alleviate oxidative stress, and strengthen immune function of broiler chickens and cultured shrimps [4,5]. In our own studies, we demonstrated that dietary C. butyricum intervention modulated serum lipid metabolism and improved both meat quality as well as fatty acid composition of Pekin ducks [6]. Therefore, C. butyricum application could promote growth performance, increase SCFA contents, and improve immune function of the intestine. However, such questions and the effects of $C$. butyricum application on the diversity of intestinal microbiota of waterfowl including for Pekin ducks has not been well researched.

Tight junctions have been considered as the primary apical components of intercellular junctions of intestinal epithelial cells [7]. Zonula occludens-1 (ZO-1) was the first intracellular component to be identified as a tight junction in 1986 [8]. Tight junctions can maintain desirable levels of permeability and barrier integrity of intestinal epithelial cells based upon examinations of both healthy and diseased treatment groups [9]. The gut plays a key role in enabling efficient absorption of nutrients due to its large surface area. The gut also includes enormous numbers and a diversity of micro-organisms, such as including opportunistic pathogens [10] which can disrupt intestinal barrier mechanisms and integrity, and induce inflammation through the release of cytokines which further disrupt the intestinal barrier. Hence, it is important for animals to have a healthy gut to limit the release of inflammatory cytokines that disrupt the intestinal barrier [11]. However, some pro-inflammatory cytokines that are known to exist in the intestine are beneficial to the overall integrity of the intestinal barrier and are important in the stimulation of release of anti-microbial substances [12]. For example, interleukin 1 beta (IL-1 $\beta$ ) is deemed to damage tight junctions but is known to be able to beneficially stimulate the expression of anti-inflammatory cytokines including IL-4 and IL-10 which strengthen integrity of the intestinal barrier [13]. However, the effects of dietary C. butyricum supplementation on tight junctions and anti-inflammatory cytokines in the intestines of Pekin ducks are unknown.

Recent increasing attention has investigated potential positive effects of applied probiotics for gut microbial communities. Several researchers have demonstrated that increased diversity of intestinal microbiota can affect various aspects of animal health including such as via regulation of the gut-liver axis and gut-brain axis [14,15]. Probiotics have been the most commonly applied alternative traditional medicines to regulate intestinal microbiota [16]. C. butyricum is thought to help optimize the intestinal microbiota and harmonize intestinal microecology via preventing the proliferation of malignant bacteria [17]. However, whether or not dietary C. butyricum supplementation can be used in a positive way to beneficially impact the diversity of intestinal microbiota and immunity in waterfowl including Pekin ducks remains unclear. In addition, short-chain fatty acids (SCFAs) known as the main metabolites of the microorganisms residing within the gastrointestinal tract can further slightly decrease the $\mathrm{pH}$ in the acidic environment of the intestine to induce an increase in the growth of probiotic bacteria, and to prevent the invasion of pathogenic bacteria in order to substantially affect gut health $[18,19]$. Thus, whether or not dietary C. butyricum could affect the concentrations of intestinal SCFAs as well as the diversity of microbial community of Pekin ducks remains to explore.

Therefore, the objectives of the present study were to assess the influences of dietary C. butyricum supplementation on growth performance, serum immunologic function, intestinal morphology, SCFAs contents, tight junction, and immune-related gene expression 
in gut, as well as the diversity of intestinal microbiota in Pekin ducks. This study will provide basic knowledge for the potential mechanism of C. butyricum in application used to modulate the gut health of Pekin ducks in the practical production.

\section{Materials and Methods}

\subsection{Ethics Approval and Consent to Participate}

All animal-based protocols conducted in this study were approved by China Agricultural University and in accordance with the Guidelines of the Animal Ethical Committee (permit no. CAU20180428-2).

\subsection{Dietary Treatments and Feeding}

We used five hundred 1-day-old male Pekin ducks (local species conventionally domesticated in Beijing, China) which were obtained from a local commercial hatchery. Ducks were raised in an experimental poultry room of China Agricultural University following normal management routines. Ducks were reared in an air-conditioned house in which could gradually modulate room temperature. Ducks were provided feed and clean drinking water ad libitum and $23 \mathrm{~h}$ of light and $1 \mathrm{~h}$ of darkness each day for $42 \mathrm{~d}$. Ducks were randomly assigned into five treatments each with 5 replicates (20 ducks/replicate). The control group (group Con) was provided a corn-soybean basal diet without antibiotic or growth promoters. Ducks in the 3 experimental groups were provided a corn-soybean basal diet with $200 \mathrm{mg} / \mathrm{kg}$ (group CB200), $400 \mathrm{mg} / \mathrm{kg}$ (group CB400), and $600 \mathrm{mg} / \mathrm{kg}$ (group CB600) C. butyricum $\left(2.0 \times 10^{9} \mathrm{cfu}\right)$. Ducks in the fifth treatment group were provided a corn-soybean basal diet with $150 \mathrm{mg} / \mathrm{kg}$ (group A150) aureomycin (powder form). The composition and nutrient levels of the basal diets were calculated to meet or exceed the requirements of the National Research Council (NRC, 1994) for the starter (1-21 d) and grower (22-42 d) periods, respectively (Table 1). The probiotic strain C. butyricum (powder form, Batch Code. 20170325003) used in this study was purchased from Beijing Shine Biology Technology Co., Ltd., Beijing, China.

The body weight (BW) of all ducks was individually weighed and feed intake was recorded for ducks from every replicate on 21 days and 42 days, respectively. Average daily feed intake (ADFI), average daily gain (ADG), and feed conversion ratio (FCR) were calculated. On day 21 and day 42, five ducks (one per replicate) of each group were randomly selected and used to obtain fresh fecal samples which were rapidly frozen in liquid nitrogen for subsequent bacterial DNA and bacterial 16S rDNA analysis.

\subsection{Serum Measurements}

Blood samples from ten ducks per group were collected from the jugular vein on day 21 and day 42 and were centrifuged at $3000 \times g$ for $10 \mathrm{~min}$; and then serum was separated and stored at $-20{ }^{\circ} \mathrm{C}$ for subsequent study. The levels of serum $\operatorname{IgA}, \operatorname{IgM}$, and IgG levels were determined using methods for ELISA commercial kits (Nanjing Jiancheng Bioengineering Co., Ltd., Nanjing, China). The concentrations of inflammatory cytokines IL-1 $\beta$, IL-4, IL-6, IL-10, and tumor necrosis factor- $\alpha$ (TNF- $\alpha$ ) were quantified with duck special cytokine/chemokine kits (Nanjing Jiancheng Bioengineering Co., Ltd., Nanjing, China). All measurements were conducted following the manufacturers' guidelines.

\subsection{Morphological Observation and Analyses}

Ten ducks from each group were euthanized using sodium pentobarbitone $(30 \mathrm{mg} / \mathrm{kg}$ BW). Distal jejunum (the dividing line between jejunum and ileum is vitelline stalk) segments were fixed in $4 \%$ formalin solution for $48 \mathrm{~h}$, dehydrated by a series of alcohol solutions, cleared in xylene, and finally were embedded in paraffin. Sections of annular of 4-5 $\mu \mathrm{m}$ thickness were stained with hematoxylin and eosin (H\&E). Intestinal tissue sample sections were scanned utilizing a Leica DMR microscope (Leica Microsystems, Wetzlar, Germany). Under the determination of a treatment-blind viewer, ten well-oriented villus and crypts were selected to evaluate the villus height and crypt depth at magnifications of 
$50 \times$ for each section using the ImagePro Plus Software. The distance of villus height was measured from the top of the villus to the villus-crypt junction; the distance of crypt depth was determined from the villus-crypt junction to the root of the crypt. Then the ratio of villus height to crypt depth $(\mathrm{V} / \mathrm{C})$ was determined.

Table 1. Composition and nutrient levels of the basal diets (air-dry basis).

\begin{tabular}{|c|c|c|}
\hline Items & $1-21 d$ & $22-42 \mathrm{~d}$ \\
\hline \multicolumn{3}{|l|}{ Ingredients (\%) } \\
\hline Corn & 56.00 & 60.24 \\
\hline Soybean meal & 32.69 & 24.67 \\
\hline Wheat middling & 5.00 & 9.00 \\
\hline Soybean oil & 2.10 & 1.80 \\
\hline Phytases & 0.02 & 0.02 \\
\hline Dicalcium phosphate & 1.00 & 1.60 \\
\hline Limestone & 1.50 & 1.20 \\
\hline DL-Methionine & 0.15 & 0.12 \\
\hline L-Lysine & 0.20 & 0.10 \\
\hline Vitamin premix ${ }^{1}$ & 0.02 & 0.02 \\
\hline Trace mineral premix ${ }^{2}$ & 0.20 & 0.20 \\
\hline $\mathrm{NaCl}$ & 0.35 & 0.30 \\
\hline Choline chloride (50\%) & 0.24 & 0.20 \\
\hline Ethoxyquin $(33 \%)$ & 0.03 & 0.03 \\
\hline Bentonite & 0.50 & 0.50 \\
\hline Total & 100 & 100 \\
\hline \multicolumn{3}{|l|}{ Nutrient levels ${ }^{3}(\%)$} \\
\hline AME $(\mathrm{MJ} / \mathrm{kg})$ & 12.31 & 12.53 \\
\hline Crude protein $(\%)$ & 19.52 & 16.83 \\
\hline Lysine $(\%)$ & 1.12 & 0.87 \\
\hline Methionine (\%) & 0.46 & 0.39 \\
\hline Calcium $(\%)$ & 0.88 & 0.89 \\
\hline Available phosphorus (\%) & 0.29 & 0.39 \\
\hline Total phosphorus (\%) & 0.54 & 0.62 \\
\hline Methionine + Cysteine (\%) & 0.79 & 0.69 \\
\hline
\end{tabular}

${ }^{1}$ The vitamin premix provided the following per kilogram of diet: vitamin $\mathrm{A}, 12,500 \mathrm{IU}$; vitamin $\mathrm{D}_{3}, 3500 \mathrm{IU}$; vitamin E, $20 \mathrm{IU}$; vitamin $\mathrm{K}_{3}, 2.65 \mathrm{mg}$; thiamin, $2.00 \mathrm{mg}$; riboflavin, $6.00 \mathrm{mg}$; pyridoxin, $3.00 \mathrm{mg}$; $\mathrm{VB}_{12}, 0.025 \mathrm{mg}$; biotin, $0.0325 \mathrm{mg}$; folic acid, $12.00 \mathrm{mg}$; pantothenic acid, $50 \mathrm{mg}$; nicotinic acid, $50.00 \mathrm{mg}$. ${ }^{2}$ The mineral premix provided the following per $\mathrm{kg}$ of diet: $\mathrm{Cu}, 6 \mathrm{mg}$; Fe, $80 \mathrm{mg}$; Zn, $40 \mathrm{mg}$; Mn, $100 \mathrm{mg}$; Se, $0.15 \mathrm{mg}$; I, $0.35 \mathrm{mg}$. ${ }^{3}$ Calculated values.

\subsection{Measurement of Intestinal SCFAs}

After animals were euthanized, we sampled fresh cecal contents (approximately $0.5 \mathrm{~g}$ ) from ten ducks per group which was diluted with a carbonate-phosphate solution. Samples were incubated using an ultrasonic bath for $30 \mathrm{~min}$ at room temperature, then were centrifuged at 13,000 rpm for $10 \mathrm{~min}$. The supernatant fraction $(500 \mu \mathrm{L})$ was collected to a new tube and then filtered through a $0.22 \mu \mathrm{m}$ filter. SCFAs analysis were conducted by using GC/MC (5975-7890A, Agilent Technologies, Inc., Santa Clare, CA, USA) and followed the guidelines described previously [20]. The areas of peaks for concentrations of acetate, propionate, and butryrate relative to 4 -methyl valeric acid were assessed to allow for the quantification of SCFAs.

\subsection{Gene Expression Measurement}

Total RNA was isolated from frozen jejunum tissue without inclusion of any remaining jejunal contents using TRIZOL Reagent (Invitrogen biotechnology Inc., Carlsbad, CA, USA) following the manufacturer's guidelines. The integrity of the extracted RNA was analyzed in $1.2 \%$ agarose electrophoresis. RNA's concentration was quantified using the Nanodrop 2000 spectrophotometer (NanoDrop Technologies, Wilmington, DE, USA) at $260 \mathrm{~nm}$; and only high-quality RNA samples were selected according to $260 / 280 \mathrm{~nm}$ ratio (1.8-2.0). Total RNA $(10 \mathrm{mg})$ was reverse transcribed to cDNA using the PrimeScriptTM RT reagent 
kit with gDNA Eraser (Takara, Dalian, China) following the manufacturer's procedures and stored at $-20^{\circ} \mathrm{C}$ for further analysis. Real-time quantitative PCR was conducted to measure the levels of expression of tight junction and immune related genes, including Muc2, ZO-1, Claudin-3, Occludin, IL4, IL10, IL6, and IL17 using the SYBR ${ }^{\circledR}$ Premix Ex TaqTM Kit (TaKaRa, Dalian, China) by an ABI QuantStudio 7 Flex Sequence Detection System (Applied Biosystems, Foster City, CA, USA). The glyceraldehyde-3-phosphate dehydrogenase (GAPDH) gene of Anas platyrhynchos was chosen as the reference internal gene and was used to normalize the initial amounts of RNA of each sample. Specificity of PCR products was measured by analysis of the resultant melting curve. All measurements were performed in triplicates for each biological repeat. The relative fold changes of intestinal gene expression were calculated using the $2^{-\Delta \Delta \mathrm{Ct}}$ method. The information of the primer sequences for the detections is listed in Table S1.

\subsection{Gut Microbiota Analysis}

Bacterial DNA was extracted from cecal contents by using the QIAamp-DNA Stool Mini Kit (Qiagen, Hilden, Germany) according to the producer's protocols. The integrity of the extracted DNA was determined in 1.0\% agarose gel electrophoresis. The concentration and quality of the extracted DNA were determined using a NanoDrop 2000 spectrophotometer (Thermo Scientific, Wilmington, DE, USA). The hypervariable 3 and 4 (V3-V4) regions of the bacterial $16 \mathrm{~S}$ rDNA gene were amplified using the extracted metagenomic DNA by PCR utilizing the specific primers of 338F and 806R (338F: $5^{\prime}-$ ACTCCTACGGGAGGCAGCA-3' and 806R: $5^{\prime}$-GGACTACHVGGGTWTCTAAT- $3^{\prime}$ ) following cycles conditions $\left(95^{\circ} \mathrm{C}\right.$ for $30 \mathrm{~s}$, followed by 27 cycles at $95{ }^{\circ} \mathrm{C}$ for $20 \mathrm{~s}, 55^{\circ} \mathrm{C}$ for $30 \mathrm{~s}$, and $72{ }^{\circ} \mathrm{C}$ for $45 \mathrm{~s}$, followed by a final extension step for $10 \mathrm{~min}$ at $72{ }^{\circ} \mathrm{C}$ ). After amplification, the PCR products were visualized on $1.2 \%$ agarose gel by electrophoresis and bands of desired sizes (approximately $300 \mathrm{bp}$ ) were purified by a QIA quick Gel Extraction Kit (Qiagen, Hilden, Germany). Amplicon libraries were normalized, pooled, and sequenced by an Illumina HiSeq 2500 PE250 Platform (Illumina, San Diego, CA, USA) at Shanghai Majorbio Bio-pharm Technology Co., Ltd. (Shanghai, China).

Sequence reads were assembled using the QIIME Software package. Raw sequence reads were quality controlled through trimming and demultiplexing steps. High-quality sequences were clustered into the same operational taxonomic units (OTUs) with a $97 \%$ similarity threshold using UPARSE (version 7.1, http:/ / drive5.com/uparse/, accessed on 12 November 2019). Representative sequences for each OTU were selected for subsequent annotation. For each representative sequence, the GreenGenes reference database was used based on the QIIME platform (http:/ / qiime.org/scripts/assign_taxonomy.html, accessed on 16 December 2019) and the RDP classifier algorithm to annotate taxonomic information. Subsequent analyses of alpha diversity index including Ace, Chao, Shannon and Sobs were calculated using QIIME platform and were visually displayed with R software. Richness and biodiversity were evaluated using measures of alpha diversity. Venn diagram was used to count the number of common and unique OTUs or individual species between groups. According to the taxonomic analyses, community structure composition of different groups at each classification level, such as at the levels of phylum and genus, were determined. Based upon the results obtained from community abundance data, microbiota differences for different groups at each classification level were detected using a rigorous statistical method.

\subsection{Statistical Analysis}

Data were analyzed using SPSS statistical software (SPSS 21.0 Inc., Chicago, IL, USA). The differences among groups in the growth performance, intestinal immune function, tight junction, intestinal morphology, and alpha diversity indices were analyzed by oneway analysis of variance (one-way ANOVA). The relative abundances of phyla and genera among the five groups were analyzed using unpaired Student's $t$-test. Significance was considered as $p<0.05$. 


\section{Results}

\subsection{Effect of C. butyricum and Aureomycin on Growth Performance of Pekin Duck}

There is no death of ducks during the whole experimental period. From the day 1 to 21, growth performance as indicated by average body weight (ABW), ADG, ADFI, and FCR among the five groups presented no significant differences $(p>0.05)$. However, from $22 \mathrm{~d}$ to $42 \mathrm{~d}$, the ABW of groups CB400 and CB600 was enhanced significantly $(p<0.05)$ in comparison with group Con. Results also revealed that the ADG of group CB400 increased significantly $(p<0.05)$ in comparison with group Con; and the FCR of group CB200 and group CB400 reduced significantly $(p<0.05)$ in comparison with results for group Con (Table 2$)$.

Table 2. Effects of Clostridium butyricum and aureomycin on growth performance of Pekin ducks ${ }^{1}$.

\begin{tabular}{cccccccc}
\hline Items $^{2}$ & $\begin{array}{c}\text { Group } \\
\text { Con }\end{array}$ & $\begin{array}{c}\text { Group } \\
\text { CB200 }\end{array}$ & $\begin{array}{c}\text { Group } \\
\text { CB400 }\end{array}$ & $\begin{array}{c}\text { Group } \\
\text { CB600 }\end{array}$ & $\begin{array}{c}\text { Group } \\
\text { A150 }\end{array}$ & SEM $^{3}$ & $p$ Value \\
\hline $1-21 \mathrm{~d}$ & & & & & & & \\
\hline ABW (kg) & 1.17 & 1.17 & 1.26 & 1.17 & 1.07 & 0.02 & 0.062 \\
ADG (g/d) & 55.98 & 54.04 & 56.21 & 53.95 & 53.42 & 0.58 & 0.439 \\
ADFI (g/d) & 90.62 & 86.36 & 85.46 & 89.24 & 86.16 & 1.23 & 0.651 \\
FCR & 1.62 & 1.61 & 1.52 & 1.66 & 1.62 & 0.03 & 0.753 \\
\hline 22-42 d & & & & & & & \\
\hline ABW (kg) & $2.88^{\mathrm{b}}$ & $2.98^{\mathrm{b}}$ & $3.18^{\mathrm{a}}$ & $3.15^{\mathrm{a}}$ & $2.94^{\mathrm{b}}$ & 0.03 & 0.009 \\
$\mathrm{ADG}(\mathrm{g} / \mathrm{d})$ & $155.09^{\mathrm{b}}$ & $157.19^{\mathrm{b}}$ & $162.75^{\mathrm{a}}$ & $155.43^{\mathrm{b}}$ & $155.31^{\mathrm{b}}$ & 0.92 & 0.028 \\
$\mathrm{ADFI}(\mathrm{g} / \mathrm{d})$ & 246.96 & $236.39^{\mathrm{b}}$ & 244.32 & 244.85 & 243.82 & 2.08 & 0.584 \\
FCR & $1.59^{\mathrm{a}}$ & $1.50^{\mathrm{b}}$ & $1.50^{\mathrm{b}}$ & $1.58^{\mathrm{a}}$ & $1.57^{\mathrm{a}}$ & 0.01 & 0.029 \\
\hline
\end{tabular}

${ }^{1}$ Means with different superscript letters indicate that there are significant differences $(p<0.05)$ between any two groups in the same row. ${ }^{2}$ ABW: average body weight, ADG: average daily gain, ADFI: average daily feed intake, FCR: feed conversion ratio, Group Con: the control group, Group A150: the aureomycin group. ${ }^{3}$ Total SEM (N =10)

\subsection{Effect of C. butyricum and Aureomycin on Immunologic Function of Pekin Duck}

On day 21, the levels of $\operatorname{IgA}, \operatorname{IgM}$, and $\operatorname{IgG}$ in serum among the five treatments presented no significant differences $(p>0.05)$. The levels of IL-4 and IL-10 in group CB600 and group A150 had increased significantly $(p<0.05)$ compared to group Con. Likewise, the levels of TNF- $\alpha$ in group A150 enhanced significantly $(p<0.05)$ in comparison with group Con. However, on day 42, the concentrations of IgA, IgM, and IgG in groups CB400 and CB600 increased significantly $(p<0.05)$ in comparison with group Con. The levels of IL-1 $\beta$ and IL-6 presented no significant differences $(p>0.05)$, whereas IL-10 levels in group CB400 were significantly elevated $(p<0.05)$, and TNF- $\alpha$ levels in group CB200 and CB400 enhanced significantly $(p<0.05)$ in comparison with group Con (Figure 1 ).

\subsection{Effects of C. butyricum and Aureomycin on Intestinal Morphology of Pekin Duck}

The gut morphology of Pekin ducks fed with C. butyricum is shown in Figure 2A. The pathological changes in the ducks in group Con were relatively mild and only a small number of epithelial cells showed dissolution, whereas the C. butyricum-treated ducks exhibited a normal appearance and complete structure of the intestinal villus. Aureomycin -treated ducks were found to have presented severe pathological changes with more broken architecture of the villus than was found in the normal group Con. On day 21, enhanced $(p<0.05)$ villus height and crypt depth in group CB600 were observed compared to group Con. The villus height to crypt depth $(\mathrm{V} / \mathrm{C})$ ratio showed no significant changes among the five treatments. However, on day 42 , greater $(p<0.05)$ villus height and $\mathrm{V} / \mathrm{C}$ values were obtained in the three $C$. butyricum-treated groups, whereas reduced $(p<0.05) \mathrm{crypt}$ depth in groups CB200 and CB400 was recorded when compared to group Con (Figure 2B). 


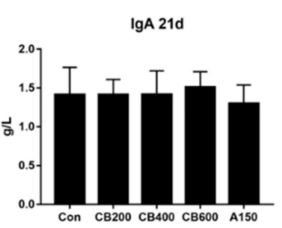

IL-6 21d

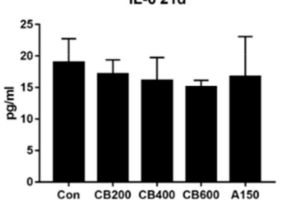

$\operatorname{lgA} 420$

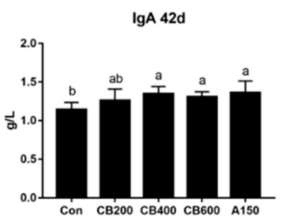

IL-6 42d

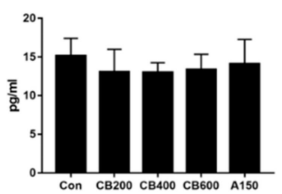

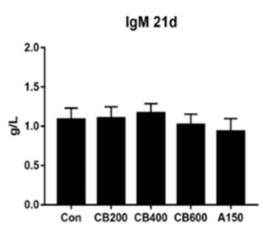

104210

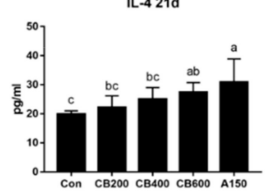

$\lg M 420$

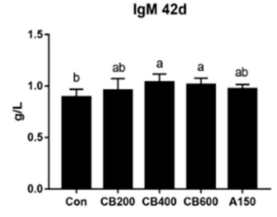

IL-4 42d

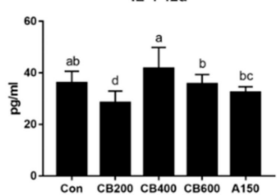

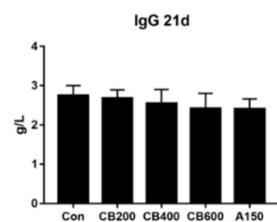

IL-10 21d

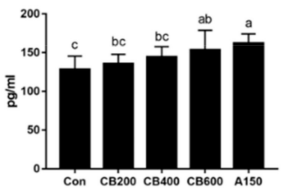

$\operatorname{lgg} 42 \mathrm{~d}$

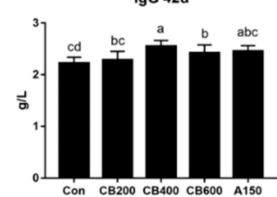

IL-10 42d

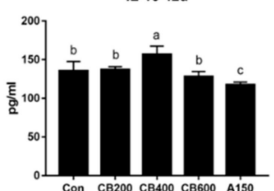

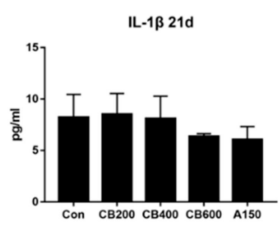

TNF-a 21d

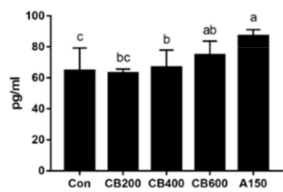

IL-1 13 42d

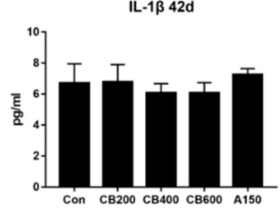

TNF- $\alpha$ 42d

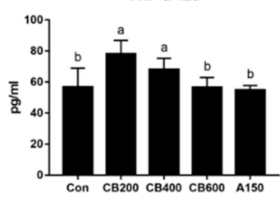

Figure 1. Effects of C. butyricum and aureomycin on serum immune parameters of Pekin ducks at $21 \mathrm{~d}$ and $42 \mathrm{~d}$, respectively $(\mathrm{N}=10$ replicates per group). The data were presented as mean $\pm \mathrm{SD}$, the superscript different letters indicate that there are significant differences $(p<0.05)$ between any two groups. Con, group Con; CB200, group CB200; CB400, group CB400; CB600, group CB600; A150, group A150.
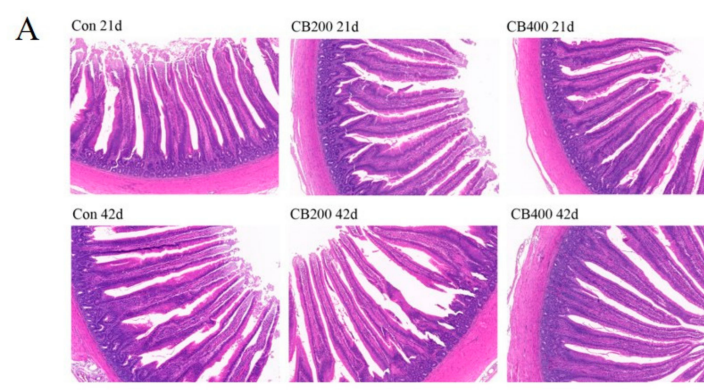

B
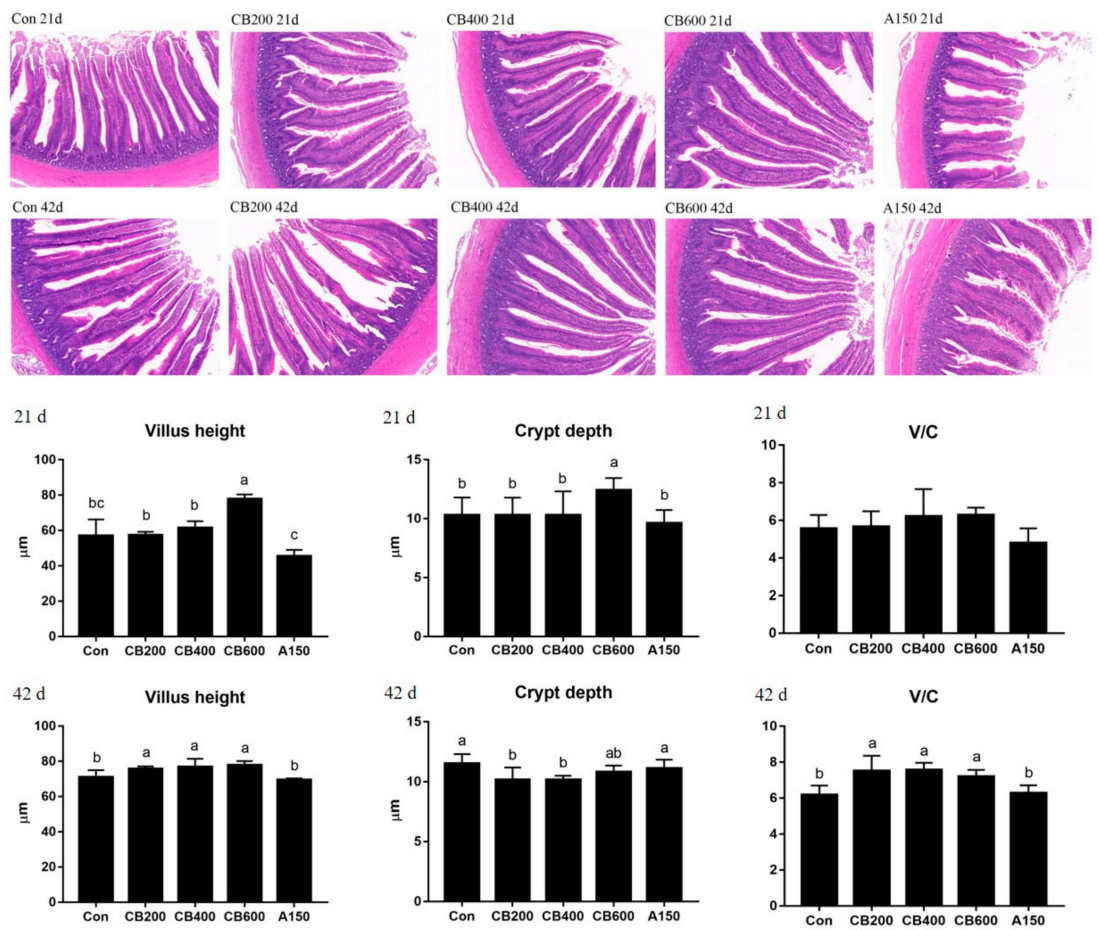

Figure 2. Effects of C. butyricum and aureomycin on intestinal morphology of Pekin ducks. (A) H\&E staining (50×) of intestinal villus. (B) The villus height and crypt depth of intestinal villus ( $\mathrm{N}=10$ replicates per group). The data were presented as mean $\pm S D$, the superscript different letters indicate that there are significant differences $(p<0.05)$ between any two groups. V/C, the ratio of villus height to crypt depth. Con, group Con; CB200, group CB200; CB400, group CB400; CB600, group CB600; A150, group A150. 


\subsection{Effect of C. butyricum and Aureomycin on Fecal SCFAs}

On day 21, the concentration of propionate in group CB400, CB600, and group A150 was lower $(p<0.05)$ whereas the concentrations of butyrate in the $C$. butyricum-treated groups and isovalerate in group CB400 were found to have increased $(p<0.05)$ in comparison with group Con. On day 42 , the concentrations of acetate in group CB400 and CB600, propionate in group CB400, butyrate in group CB400 and A150, iso-butyrate and iso-valerate in all groups increased $(p<0.05)$ significantly in comparison with group Con (Table 3$)$.

Table 3. Influence of Clostridium butyricum and aureomycin supplementation on short-chain fatty acids concentrations of Pekin ducks ${ }^{1}(\mu \mathrm{g} / \mathrm{g})$.

\begin{tabular}{|c|c|c|c|c|c|c|c|}
\hline Items & $\begin{array}{l}\text { Group } \\
\text { Con }\end{array}$ & $\begin{array}{l}\text { Group } \\
\text { CB200 }\end{array}$ & $\begin{array}{l}\text { Group } \\
\text { CB400 }\end{array}$ & $\begin{array}{l}\text { Group } \\
\text { CB600 }\end{array}$ & $\begin{array}{c}\text { Group } \\
\text { A150 }\end{array}$ & $S E M^{2}$ & $p$ Value \\
\hline \multicolumn{8}{|l|}{21 day } \\
\hline Acetate & 526.61 & 517.34 & 540.61 & 527.64 & 504.90 & 4.60 & 0.136 \\
\hline Propionate & $283.37^{\mathrm{a}}$ & $270.80^{\mathrm{ac}}$ & $245.50^{b c}$ & $251.82^{b c}$ & $211.23^{\mathrm{d}}$ & 7.54 & 0.004 \\
\hline Iso-butyrate & 122.81 & 128.40 & 137.27 & 128.32 & 126.84 & 1.67 & 0.056 \\
\hline Butyrate & $1360.59^{d}$ & $1513.37^{c}$ & $1560.94^{\mathrm{a}}$ & $1556.94^{\mathrm{ab}}$ & $1539.56^{\mathrm{ab}}$ & 20.23 & $<0.001$ \\
\hline Iso-valerate & $42.74^{c}$ & $51.38^{\mathrm{a}}$ & $49.65^{a b}$ & $45.98^{\mathrm{bc}}$ & $46.14^{\mathrm{bc}}$ & 0.95 & 0.006 \\
\hline Valerate & 62.60 & 60.18 & 61.08 & 57.48 & 58.97 & 1.01 & 0.619 \\
\hline \multicolumn{8}{|l|}{42 day } \\
\hline Acetate & $647.54^{b}$ & $617.41^{b}$ & $780.75^{a}$ & $777.65^{a}$ & $681.57^{b}$ & 21.21 & 0.037 \\
\hline Propionate & $283.38^{a b}$ & $297.46^{a b}$ & $352.17^{a}$ & $255.15^{\mathrm{ab}}$ & $218.89^{b}$ & 4.72 & 0.022 \\
\hline Iso-butyrate & $124.48^{c}$ & $145.06^{\mathrm{ab}}$ & $157.50 \mathrm{ab}$ & $165.00^{a}$ & $144.84^{\mathrm{b}}$ & 4.40 & 0.011 \\
\hline Butyrate & $1773.96^{b c}$ & $1909.93^{\mathrm{ab}}$ & $2070.21^{a}$ & $2000.27^{a}$ & $1736.23 \mathrm{bc}$ & 39.73 & 0.006 \\
\hline Iso-valerate & $48.74^{\mathrm{b}}$ & $74.71^{\mathrm{a}}$ & $87.98^{a}$ & $89.31^{\mathrm{a}}$ & $89.41^{\mathrm{a}}$ & 5.21 & 0.024 \\
\hline Valerate & 85.93 & 126.84 & 124.41 & 137.48 & 112.30 & 6.96 & 0.152 \\
\hline
\end{tabular}

${ }^{1}$ Means with different superscript letters indicate that there are significant differences $(p<0.05)$ between any two groups in the same row, Group Con: the control group, Group A150: the aureomycin group. ${ }^{2}$ Total SEM (N = 10)

\subsection{Expression of Tight Junction and Immune Related-Genes in Gut}

On day 21, in comparison with group Con, the relative levels of mRNA expression of tight junction protein related-gene including Muc2 in group CB200 and CB400, ZO-1 in group CB400, CB600, and A150, Claudin-3 in group CB400 and A150, and Occludin in group CB400, CB600, and A150, were all enhanced $(p<0.05)$ (Figure 3 ); whereas the relative levels of mRNA expression of immune-related gene such as pro-inflammatory cytokine genes (IL10) increased $(p<0.05)$ and anti-inflammatory cytokine genes (IL6 and IL17) decreased $(p>0.05)$ in the three C. butyricum-treated groups (Figure 4). On day 42, in comparison with group Con, the relative levels of mRNA expression of tight junction protein related-gene (Muc2, ZO-1, Claudin-3, and Occludin) and immune-related gene (proinflammatory cytokine IL4 and IL10) were also increased $(p<0.05)$ in the three $C$. butyricum -treated groups, whereas the levels of mRNA expression of immune-related gene (antiinflammatory cytokine IL6 and IL17) were reduced $(p>0.05)$. The mRNA expression levels of tight junction proteins and anti-inflammatory cytokine related-genes were the highest in the group CB400.

\subsection{Quality of Sequencing Data}

$16 \mathrm{~S}$ rDNA gene sequencing of fecal samples was performed to evaluate changes of intestinal bacteria after dietary C. butyricum intervention. A total of 1,235,064 and $1,684,981$ clean sequences were acquired from fecal samples after quality control, size filtering, and chimera removal at $21 \mathrm{~d}$ and $42 \mathrm{~d}$, respectively (Supplementary file 1 ). The average sequence length was 429 base pairs (bp) with a range of $250 \mathrm{bp}$ to $539 \mathrm{bp}$ at $21 \mathrm{~d}$, and 436 base pairs (bp) with a range of $228 \mathrm{bp}$ to $532 \mathrm{bp}$ at $42 \mathrm{~d}$ (Supplementary file 2). The total operational taxonomic units (OTU) numbers which were identified according to $>97 \%$ sequencing similarity were 390 OTUs and 547 OTUs detected in fecal samples on day 21 and day 42 , respectively. The number of OTUs in each group is presented below the Venn diagram on day 21 and day 42, respectively (Figure 5). 


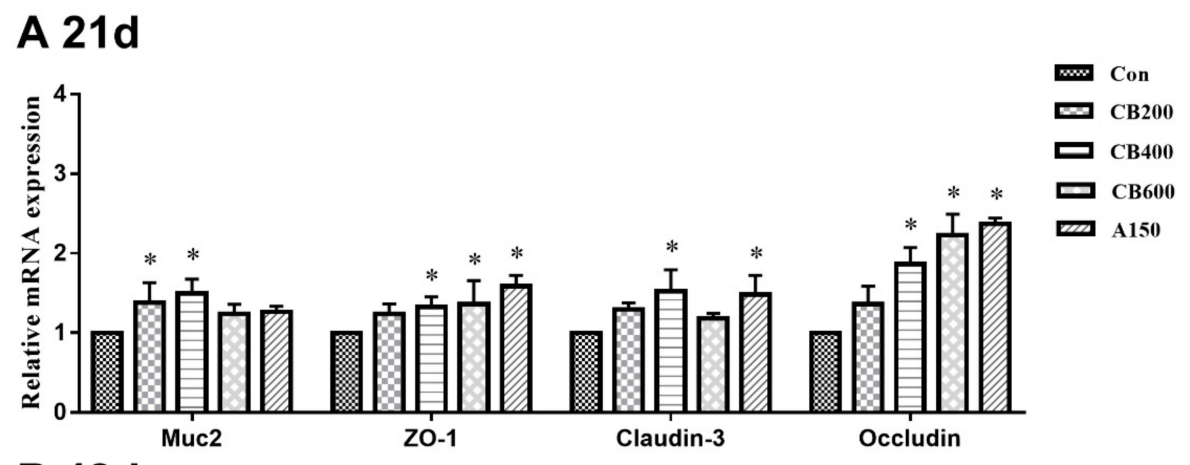

\section{B 42d}

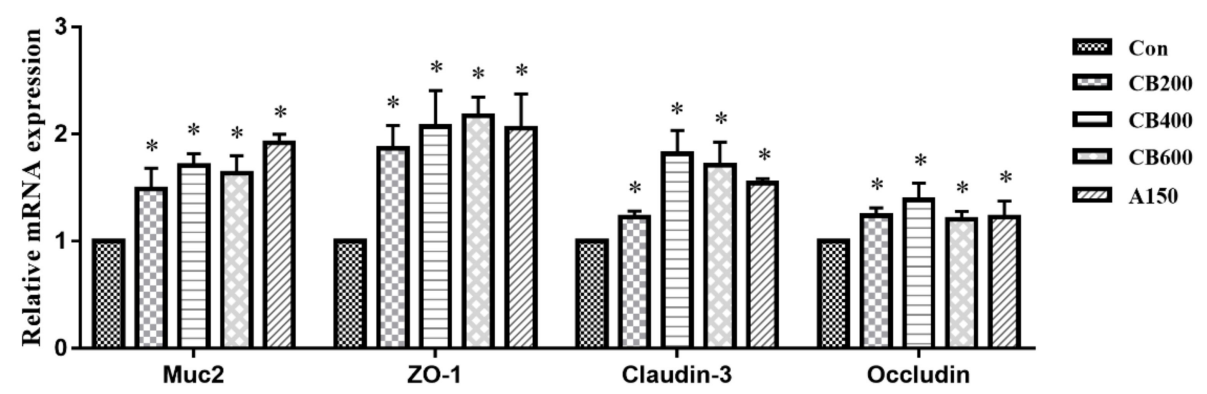

Figure 3. Effects of C. butyricum and aureomycin on relative mRNA expression of tight junction protein genes of intestine in Pekin ducks ( $\mathrm{N}=10$ replicates per group). (A) The relative mRNA expression of tight junction protein genes at $21 \mathrm{~d}$; (B) The relative mRNA expression of tight junction protein genes at $42 \mathrm{~d}$. The superscript * indicate that there are significant differences $(p<0.05)$ compared with the control group. Con, group Con; CB200, group CB200; CB400, group CB400; CB600, group CB600; A150, group A150.

\section{A 21d}
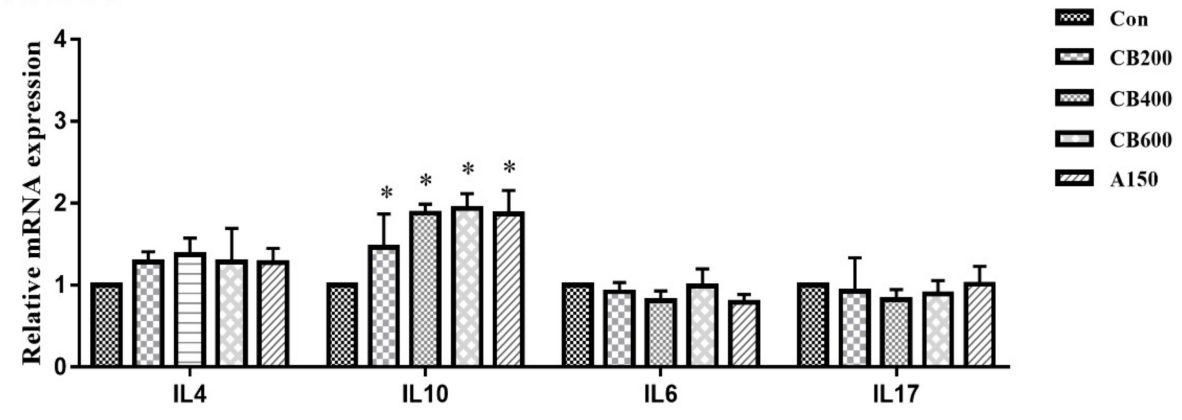

B 42d

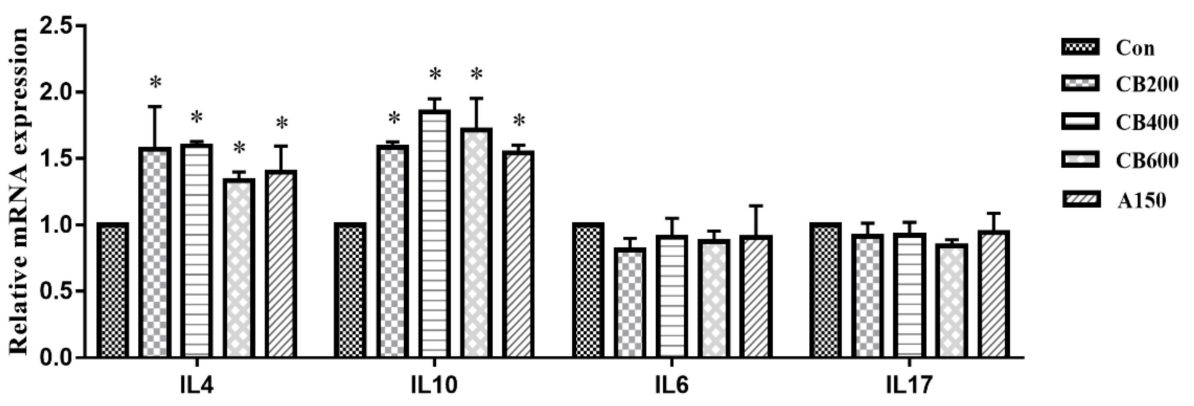

Figure 4. Effects of C. butyricum and aureomycin on relative mRNA expression of inflammatory cytokine genes of intestine in Pekin ducks ( $\mathrm{N}=10$ replicates per group). (A) The relative mRNA expression of inflammatory cytokine genes at $21 \mathrm{~d}$; (B) The relative mRNA expression of inflammatory cytokine genes at $42 \mathrm{~d}$. The superscript * indicate that there are significant differences $(p<0.05)$ compared with the control group. Con, group Con; CB200, group CB200; CB400, group CB400; CB600, group CB600; A150, group A150. 

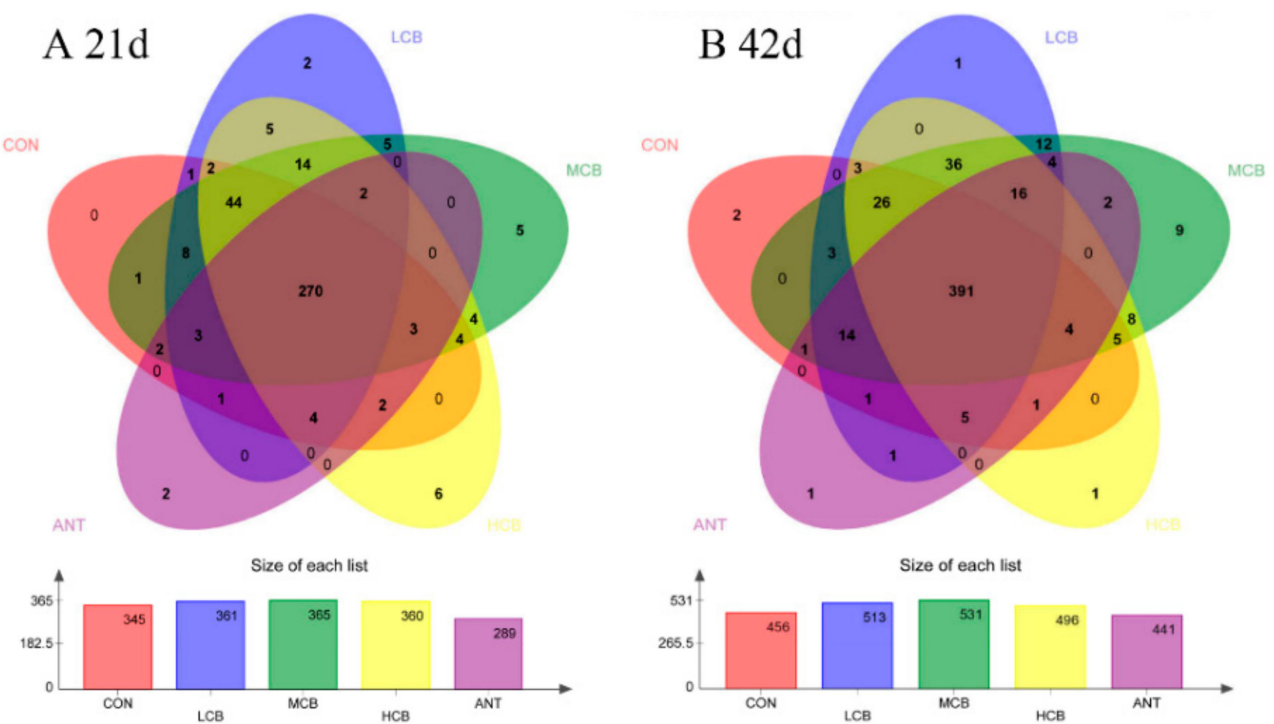

C 21d

D $42 \mathrm{~d}$
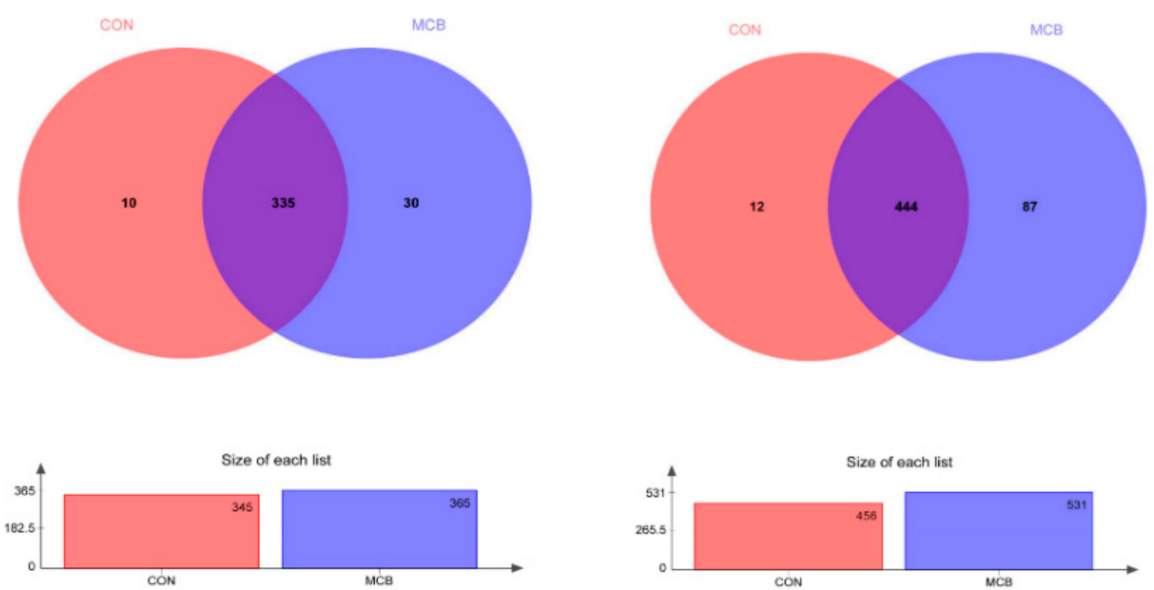

Figure 5. Venn diagram for intestinal microbiota of Pekin ducks $(\mathrm{N}=5)$. Each circle represents a group of samples. The numbers of common bacterial operational taxonomic units (OTUs) are showed in the overlapping part between different circles, while the numbers in the non-overlapping part between different circles represent the number of their respectively unique OTUs in each group. (A), Venn diagram of five groups at $21 \mathrm{~d}$; (B), Venn diagram of group A and group C at $21 \mathrm{~d}$; (C), Venn diagram of five groups at $42 \mathrm{~d}$; (D), Venn diagram of group A and group C at $42 \mathrm{~d}$; CON, group Con; LCB, group CB200; MCB, group CB400; HCB, group CB600; ANT, group A150.

The Ace index, Chao index, Shannon index, and Sobs index were used to explore the influences of $C$. butyricum on bacterial abundance and diversity. In comparison with group Con (named as CON in Figure 6), the Ace index, Chao index, and Shannon index in C. butyricum-treated groups showed no significant differences $(p>0.05)$ at $21 \mathrm{~d}$. The Sobs index in the group CB400 (named as MCB in Figure 6) increased while decreased in the group A150 (named as ANT in Figure 6) significantly $(p<0.05)$ compared with the control group (Figure 6). However, at $42 \mathrm{~d}$, in comparison with the CON, the Ace index in group MCB and group CB600 (named as HCB in Figure 6), the Chao index in group $\mathrm{MCB}$, the Shannon indexes in group $\mathrm{MCB}$ and $\mathrm{HCB}$, and the Sobs indexes in three C. butyricum-treated groups increased significantly $(p<0.05)$ (Figure 6). 

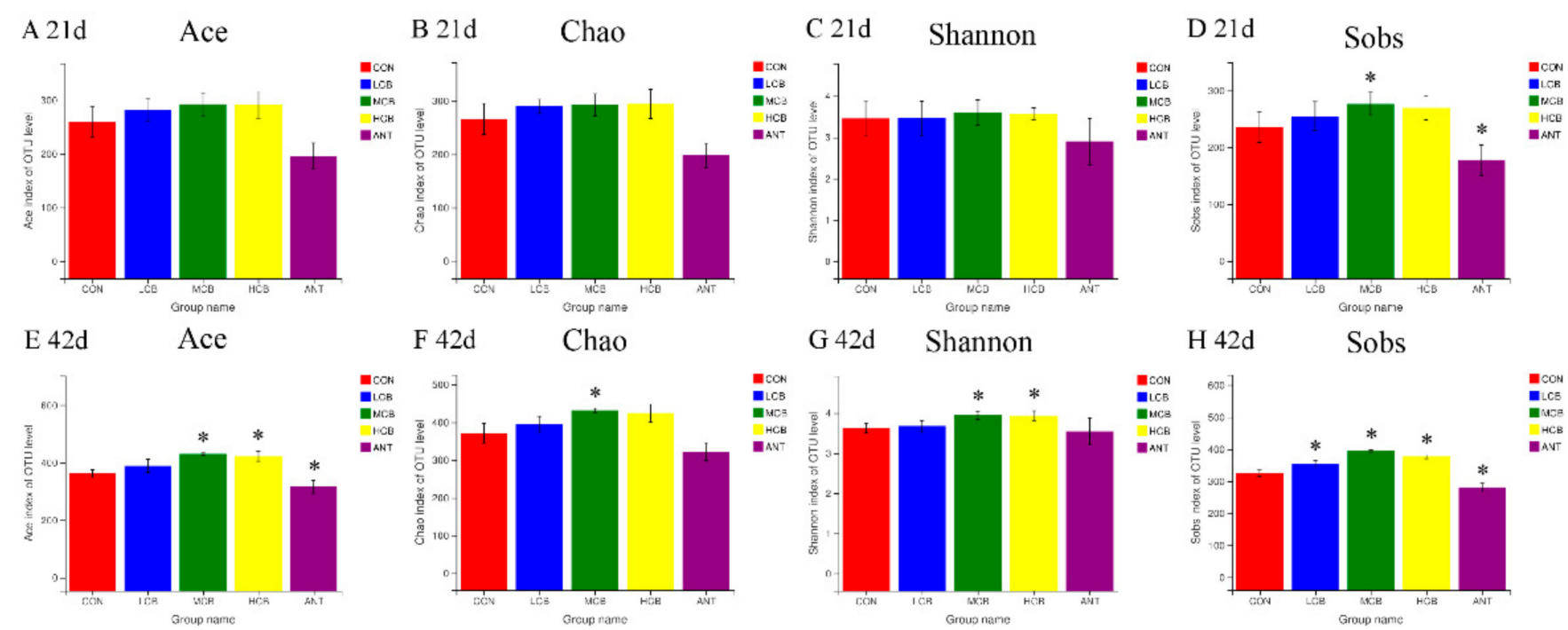

Figure 6. Estimators of $\alpha$-diversity indices in ceca of Pekin ducks in the five groups at different timepoint. (A), Ace index at $21 \mathrm{~d} ;(\mathbf{B})$, Chao index at $21 \mathrm{~d} ;(\mathbf{C})$, Shannon index at $21 \mathrm{~d}$; (D), Sobs index at $42 \mathrm{~d} ;($ E), Ace index at $42 \mathrm{~d} ;(\mathbf{F})$, Chao index at $42 \mathrm{~d} ;(\mathrm{G})$, Shannon index at $42 \mathrm{~d} ;(\mathbf{H})$, Sobs index at $42 \mathrm{~d}$. The superscript * indicates that there are significant differences $(p<0.05)$ compared with the control group. CON, group Con; LCB, group CB200; MCB, group CB400; HCB, group CB600; ANT, group A150.

\subsection{Effect of C. butyricum and Aureomycin on Relative Abundances of Species Structure and Community Composition}

A total of eight different bacterial phyla were identified on $21 \mathrm{~d}$ (Figure 7A). Firmicutes, Bacteroidetes, Fusobacteria, and Tenericutes were the four-dominant phylum (relative abundance $>1 \%$ ) in the five clustered groups. Firmicutes was the most abundant phyla in all groups compared to the other phyla, especially in group CB400, as the relative abundance of Firmicutes accounted for $92.27 \%$ in group Con, $92.65 \%$ in group CB200, $93.24 \%$ in group CB400, $90.34 \%$ in group CB600, and $80.07 \%$ in group A150, respectively. The relative abundance of Bacteroidetes was found to be $4.04 \%$ in group Con, $2.47 \%$ in group CB200, $2.87 \%$ in group CB400, $2.75 \%$ in group CB600, and $6.59 \%$ in group $\mathrm{A} 150$, respectively. The ratio of Firmicutes/Bacteroidetes was found to be 22.84, 37.51, 32.49, 32.85, and 12.15 for the same groups above, respectively. At the family level, Lachnospiraceae, Ruminococcaceae, Erysipelotrichaceae, and Streptococcaceae were the primary intestinal bacteria in all of the groups assessed (Figure 7B). The relative abundance of Lachnospiraceae was enriched in the groups Con, CB200, CB600, and A150 (relative abundance $=43.00-55.06 \%$ ) but were apparently weakened in the group CB400 (relative abundance $=23.58 \%$ ). The relative abundance of Ruminococcaceae was enriched in group CB400 (relative abundance $=23.30 \%$ ) whereas weakened in the other four groups. However, a total of twelve different bacterial phyla were identified on $42 \mathrm{~d}$ (Figure 7C). Firmicutes, Bacteroidetes, Proteobacteria, Actinobacteria, and Spirochaetae were the five dominant phyla (relative abundance $>1 \%$ ) in the five clustered groups. Firmicutes and Bacteroidetes were the most abundant two phyla in all groups compared to the other phyla. The relative abundance of Firmicutes was $65.21 \%$ in group Con, $52.92 \%$ in group CB200, $55.85 \%$ in group CB $400,56.64 \%$ in group CB600, and $57.67 \%$ in group A150, respectively. The relative abundance of Bacteroidetes was $28.90 \%$ in group Con, $42.72 \%$ in group CB200, $38.80 \%$ in group CB400, $40.52 \%$ in group CB600, and $40.17 \%$ in group A150, respectively. The ratio of Firmicutes/Bacteroidetes was found to be 2.26, 1.24, 1.43, 1.40, and 1.44 for the same groups above, respectively. At the family level, Bacteroidaceae, Lachnospiraceae, Prevotellaceae, Ruminococcaceae, Veillonellaceae, and Acidaminococcaceae were the main intestinal bacteria in all the five groups assessed (Figure 7D). The diversity of species structure and community composition was found to have demonstrated an obvious increased on $42 \mathrm{~d}$ at family level. 

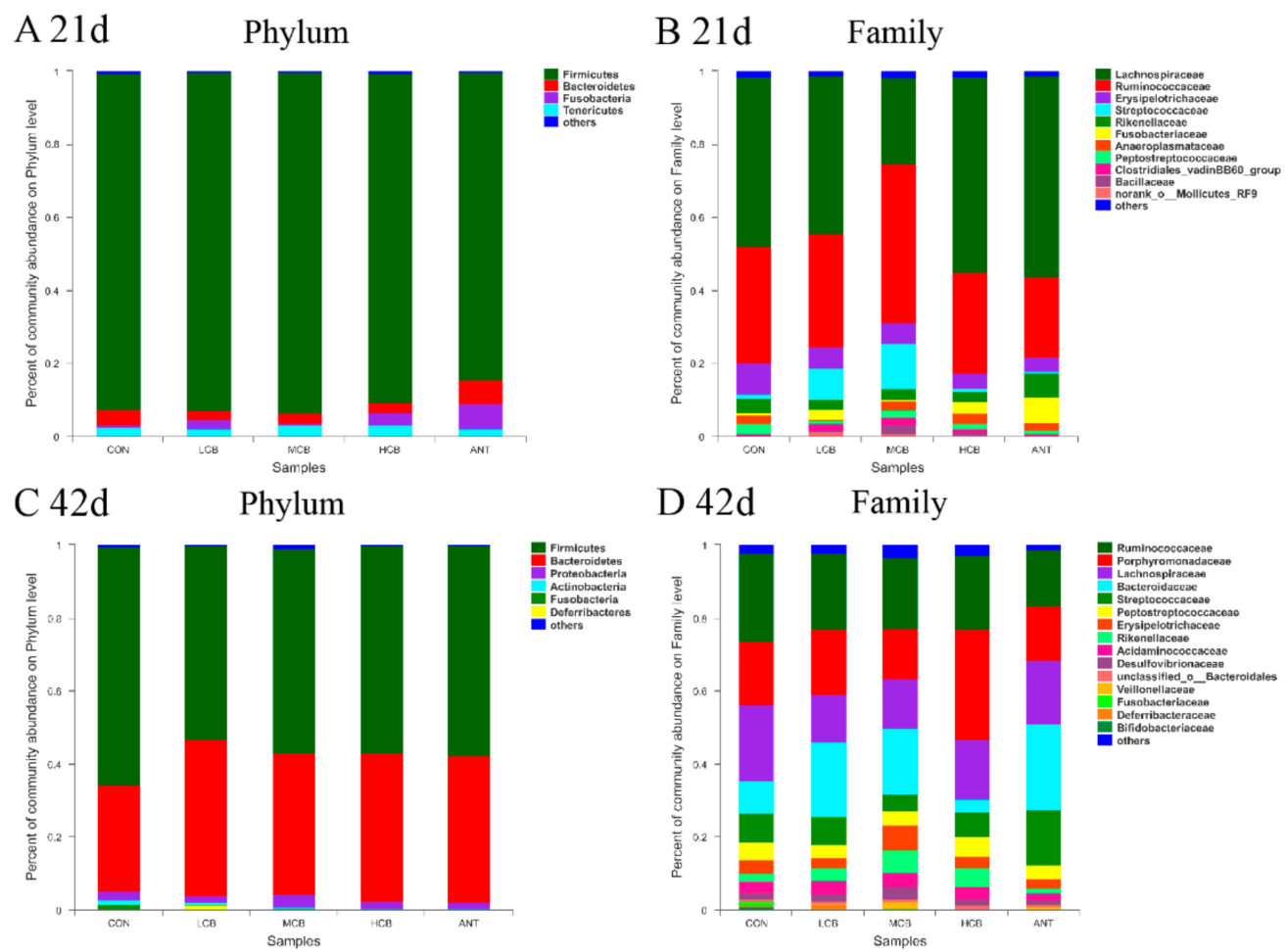

Figure 7. Relative abundance (\%) and composition evaluated at the phylum and family levels of the intestinal microbiome of Pekin ducks in the five groups at different timepoint. (A), $21 \mathrm{~d}$ at phylum level; (B), $21 \mathrm{~d}$ at family level; (C), $42 \mathrm{~d}$ at phylum level; (D), $42 \mathrm{~d}$ at family level. CON, group Con; LCB, group CB200; MCB, group CB400; HCB, group CB600; ANT, group A150.

\section{Discussion}

Numerous studies have demonstrated that an appropriate level of C. butyricum administration can increase growth performance in different kinds of animals owing to C. butyricum could enhance digestibility of nutrients and energy [5,21,22]. In our study, $400 \mathrm{mg} / \mathrm{kg}$ C. butyricum can increase ABW of Pekin ducks at day 42, but ADG, ADFI, and FCR were not significantly influenced in the first 21 days of feeding trial. Only ADG and FCR were increased over the second 21 days of feeding trial. These results suggest that positive effects of $C$. butyricum on animal growth were in time-dependent relationship. Our findings are consistent with the above studies.

The strong intestine barrier depends on structural integrity, tight junction proteins, and a stable microbiome [9]. In fact, one of the primary defense mechanisms against invasion is the intestinal epithelial barrier [23]. In order to improve human and animal health, nutritional or pharmacological interventions have been designed to enhance the intestinal epithelial barrier, and such interventions are increasingly being actively sought after by researchers and industry. These positive effects induced by the application of probiotics act as an important role in regulating growth performance and gut health of poultry [24]. Dietary interventions such as probiotics have been found to be able to improve intestinal barrier function via inhibiting inflammation or directly impacting barrier function [25]. Thus, we sought to examine the roles of C. butyricum on helping to regulate intestinal immunity and intestinal barrier function as well as the morphological structure of intestinal componentry. To support the above view, an improved intestinal morphology in jejunum was observed in Pekin duck, as evidenced by a significant increase in villus height after dietary $400 \mathrm{mg} / \mathrm{kg}$ or $600 \mathrm{mg} / \mathrm{kg}$ C. butyricum supplementation. In some previous reports, increased intestinal morphological structure was observed in broilers and pigs after probiotic supplementation, which is consistent with ours [21,26,27]. Intestinal villus, as the interface and the link of the host with the environment, plays 
an important role in maintaining efficient absorption and intestinal barrier integrity [28]. Hence, the increased intestinal morphology manifested an enhancement of intestinal barrier integrity and absorption ability of available nutrients of Pekin ducks in response to C. butyricum addition, suggesting that there was better growth performance. As is wellknown, Muc2, ZO-1, Occludin and Claudin-1 are the four primary tight junction proteins to maintain the intestinal barrier function in the small intestine [10]. In previous reports, dietary intervention or inflammation reaction can affect the tight junction proteins [29] and enhanced levels of $m R N A$ expression of tight junction proteins including Occludin and ZO-1 can reduce inflammatory stimulus [30]. Our results accordingly demonstrated that C. butyricum addition improved the intestinal epithelial barrier function by increasing the mRNA levels of tight junction protein expression. The inflammatory stimuli and other endogenous cytokines were directly to have been directly weakened due to increases in the expression and localization of tight junction proteins which ultimately reinforced the intestinal barrier.

SCFAs are known as the main end products metabolized by microorganisms in the guts of animals [31]. Butyrate has been reported as one of the major SCFAs, and has accordingly been denoted to have a wide range of biological effects. For example, butyrate can inhibit the growth of harmful bacteria, regulate immunity, and change the community composition of gut microbiota [32]. We found that the contents of butyrate in three C. butyricum-treated groups at $21 \mathrm{~d}$ and in $400 \mathrm{mg} / \mathrm{kg}$ and $600 \mathrm{mg} / \mathrm{kg}$ C. butyricum-treated groups at $42 \mathrm{~d}$ enhanced significantly. Dietary supplementation of butyrate in poultry can improve growth performance, carcass yield, intestinal immunity function, and inhibit infection of Salmonella enteritidis [33]. Likewise, butyrate was proved to decrease the expression of proinflammatory (IL-1 $\beta$, IL-6, and IFN- $\alpha$ ) and increase the expression of anti-inflammatory cytokine (IL-10) genes [34]. These previous researches are in agreement with our results.

Cytokines play a vital role in maintaining the host immune system and intestinal immunity to resist pathogen invasion and modulating the immunity response appropriately [35]. The immune system of poultry gastrointestinal tract can be affected by the probiotics intervention [36]. Probiotics intervention beneficially reinforces the intestinal immunity accompanied by decreased pro-inflammatory cytokines, such as IL-1 $\beta$, IL-6, and TNF- $\alpha$ and increased anti-inflammatory cytokines, such as IL-4, and IL-10. Increasing the production of anti-inflammatory cytokines might have undiscovered additional underlying advantages in inhibiting intestinal mucosal inflammation [37]. C. butyricum can bring a series of microbial activities that stimulate innate immune cells by enhancing immunity response and reducing the production of pro-inflammatory cytokines (IL-1 $\beta$, IL-6, TNF$\alpha$ and so on) [38]. In our study, the contents of $\operatorname{IgA}, \operatorname{IgG}$, and IgM were not affected by C. butyricum treatment at $21 \mathrm{~d}$ while increased after $400 \mathrm{mg} / \mathrm{kg}$ and $600 \mathrm{mg} / \mathrm{kg}$ C. butyricum intervention at $42 \mathrm{~d}$. IL-4 and IL-10, which can inhibit the production of pro-inflammatory cytokines, are generated by Th2 cells via C. butyricum-stimulated signal transduction of GPR109a [39]. Notably, $400 \mathrm{mg} / \mathrm{kg}$ C. butyricum supplementation had enhanced the production of IL-10 both in serum at $21 \mathrm{~d}$ and gut at $21 \mathrm{~d}$ and $42 \mathrm{~d}$ suggesting that $C$. butyricum could improve the response of anti-inflammatory cytokines to some extent in Pekin ducks. IL-10 acted an important role in maintaining the production of mucins in intestinal goblet cells, and the mucins can positively reinforce intestinal barrier function [40]. Thereby, the improvement of IL-10 production implied that $400 \mathrm{mg} / \mathrm{kg}$ C. butyricum likely played a role in the initial protective barrier function of the intestine.

The influences of gut microbiota on growth performance and body health in poultry, for instance digestion and nutrient absorption, immune response, as well as protection of animals against pathogens, have become increasingly noticeable [41]. The diversity of intestinal microbiota is the foundation for digestion and nutrient absorption, the intestinal physiological functions, and the development of the immune system [42]. Previous studies demonstrated that probiotics can regulate intestine microbiota via a competitive exclusion process wherein decreasing the growth of harmful bacteria and increasing the growth of favorable bacteria [43-45]. In our current study, an increased richness and biodiversity 
were found in the intestinal microbial community when Pekin ducks were supplemented with $400 \mathrm{mg} / \mathrm{kg} \mathrm{C}$. butyricum via OTUs determinations and $\alpha$-diversity analysis. Further, obvious differences were noted between the C. butyricum-treated groups and the control group, suggesting that $C$. butyricum supplementation, especially at a moderate dose $(400 \mathrm{mg} / \mathrm{kg})$, enhanced the abundance and diversity of intestine microbiota in Pekin ducks. The Venn diagram based on OTUs analysis also supported the above-mentioned results, which revealed that the ducks fed with $400 \mathrm{mg} / \mathrm{kg}$ dietary C. butyricum displayed the largest number of unique bacterial OTUs in the intestinal microbiota.

The Firmicutes and Bacteroidetes dominated the intestinal composition of Pekin ducks at all growth stages which is consistent with previous studies of other animals [27,46]. The results of taxonomic composition comparisons demonstrated an enrichment of the phyla Firmicutes and Bacteroidetes in the intestine after C. butyricum addition. A great number of studies have confirmed probiotic effects of Bacteroidetes and Firmicutes, which seemingly act as important players in polysaccharide decomposition, and consequently, contribute to improved nutrient utilization, increased immune system development, and help to maintain intestinal microecological balance $[47,48]$. Notably, the relative abundance of Firmicutes and feed utilization presented a positive correlation as we found that the decreased feed conversion ratio of Pekin ducks was accompanied by the enhanced relative abundance of Firmicutes. On day 21, at phyla level, approximately $90 \%$ of the relative abundant phyla were Firmicutes in the intestine of Pekin ducks fed diet with $400 \mathrm{mg} / \mathrm{kg}$ C. butyricum. This finding may be associated with those younger animals need more intestinal bacteria members belonged to Firmicutes for digestion and absorbance of nutrition, for instant Lachnospiraceae, Ruminococcaceae, Erysipelotrichaceae, and Streptococcaceae. As Pekin ducks aged, the percentages of Firmicutes phyla were reduced, while the population of Bacteroidetes phyla was increased in the gut with continuous dietary C. butyricum intervention. Therefore, the reduction in Firmicutes phyla and the increase in Bacteroidetes phyla played a critical role in improving feed efficiency and intestinal structure of ducks with $400 \mathrm{mg} / \mathrm{kg} \mathrm{C}$. butyricum supplementation. These results were in agreement with a previous report in which C. butyricum can enhance the relative abundance of Bacteroidetes in weaned pigs [49]. In another study, the dominant functions of intestine microbiota, such as Firmicutes and Bacteroidetes as well as their interactions, can support the need to further explore a special probiotic that can be best used and can guide us to apply the special probiotics to achieve the anticipated goals for keepers of livestock and breeders.

In addition to our findings, $400 \mathrm{mg} / \mathrm{kg}$ C. butyricum supplementation presented better regulations on the relative abundance of microbiota communities at the family level form $21 \mathrm{~d}$ to $42 \mathrm{~d}$ than $200 \mathrm{mg} / \mathrm{kg}$ or $600 \mathrm{mg} / \mathrm{kg}$ C. butyricum supplementation. Bacteroidaceae was identified as a beneficial microbe that regulated gut health and helped to absorb polysaccharide from feed which can stimulate the proliferation of Lactobacillus [33]. Increased abundances of Bacteroidaceae and Ruminococcaceae were identified with $400 \mathrm{mg} / \mathrm{kg}$ C. butyricum intervention. Ruminococcaceae was proved to be involved with the degradation of cellulose and hemicelluloses in the intestine [50]. Hence, it can be assumed that the enhanced abundance of Bacteroidaceae may be associated with improved intestinal immunity of Pekin ducks. Thus, it could be speculated that $400 \mathrm{mg} / \mathrm{kg}$ C. butyricum addition better modulates the composition and abundance of favorable and pernicious bacteria, which can improve the intestinal health of ducks.

\section{Conclusions}

In summary, the current study demonstrated that dietary supplementation with C. butyricum improved growth performance, host immunity, the concentration of SCFAs, and intestinal tight junctions as well as enhanced abundance and diversity of gut microbiota of Pekin ducks, and the optimum dosage of C. butyricum is $400 \mathrm{mg} / \mathrm{kg}$ for ducks in the practical production. Further studies can focus on the development of new intervention strategies of probiotics to modulate the gut microbiota to improve the growth performance and productivity of waterfowl. 
Supplementary Materials: The following are available online at https: / www.mdpi.com/article/ 10.3390/ani11092514/s1, Supplementary file 1: Information of clean reads, Supplementary file 2: Quality control data of each sample, Table S1: Information of primers used in qRT-PCR.

Author Contributions: Conceptualization, Y.L. and Z.X.; investigation, Y.L. and C.L.; resources, K.A.; data curation, Y.L. and X.G.; writing-original draft preparation, Y.L.; writing-review and editing, Y.L., C.L. and K.A.; supervision, X.G.; project administration, Y.L. and X.G.; funding acquisition, Z.X. All authors have read and agreed to the published version of the manuscript.

Funding: This research was supported by the System for Poultry Production Technology, Beijing Innovation Research Team of Modern Agriculture (BAIC04-2021) and Shandong Modern Agricultural Technology \& Industry System (SDAIT-11-13).

Institutional Review Board Statement: All animal-based protocols conducted in this study were approved by China Agricultural University and in accordance with the Guidelines of the Animal Ethical Committee (permit code. CAU20180428-2).

Informed Consent Statement: Not applicable.

Data Availability Statement: Data sharing is not applicable.

Conflicts of Interest: The authors declare that they have no competing interests.

\section{References}

1. Liang, J.; Kou, S.; Chen, C.; Raza, S.H.A.; Wang, S.; Ma, X.; Zhang, W.J.; Nie, C. Effects of Clostridium butyricum on growth performance, metabonomics and intestinal microbial differences of weaned piglets. BMC Microbiol. 2021, 21, 85. [CrossRef]

2. Zhao, X.; Ding, X.; Yang, Z.; Shen, Y.; Zhang, S.; Shi, S. Effects of Clostridium butyricum on breast muscle lipid metabolism of broilers. Ital. J. Anim. Sci. 2018, 17, 1010-1020. [CrossRef]

3. Hagihara, M.; Yamashita, R.; Matsumoto, A.; Mori, T.; Kuroki, Y.; Kudo, H.; Oka, K.; Takahashi, M.; Nonogaki, T.; Yamagishi, Y.; et al. The impact of Clostridium butyricum MIYAIRI 588 on the murine gut microbiome and colonic tissue. Anaerobe 2018, 54, 8-18. [CrossRef] [PubMed]

4. Liao, X.; Wu, R.; Ma, G.; Zhao, L.; Zheng, Z.; Zhang, R. Effects of Clostridium butyricum on antioxidant properties, meat quality and fatty acid composition of broiler birds. Lipids Health Dis. 2015, 14, 36. [CrossRef] [PubMed]

5. Duan, Y.; Wang, Y.; Dong, H.; Ding, X.; Liu, Q.; Li, H.; Zhang, J.; Xiong, D. Changes in the Intestine Microbial, Digestive, and Immune-Related Genes of Litopenaeus vannamei in Response to Dietary Probiotic Clostridium butyricum Supplementation. Front. Microbiol. 2018, 9, 2191. [CrossRef] [PubMed]

6. Liu, Y.; Li, Y.; Feng, X.; Wang, Z.; Xia, Z. Dietary supplementation with Clostridium butyricum modulates serum lipid metabolism, meat quality, and the amino acid and fatty acid composition of Peking ducks. Poult. Sci. 2018, 97, 3218-3229. [CrossRef]

7. Fu, J.; Wang, T.; Xiao, X.; Cheng, Y.; Wang, F.; Jin, M.; Wang, Y.; Zong, X. Clostridium Butyricum ZJU-F1 Benefits the Intestinal Barrier Function and Immune Response Associated with Its Modulation of Gut Microbiota in Weaned Piglets. Cells 2021, 10, 527. [CrossRef] [PubMed]

8. Stevenson, B.R.; Siliciano, J.D.; Mooseker, M.S.; Goodenough, D.A. Identification of ZO-1: A high molecular weight polypeptide associated with the tight junction (zonula occludens) in a variety of epithelia. J. Cell. Biol. 1986, 103, 755-766. [CrossRef]

9. Wang, Y.; Tong, J.; Chang, B.; Wang, B.; Zhang, D.; Wang, B. Effects of alcohol on intestinal epithelial barrier permeability and expression of tight junction-associated proteins. Mol. Med. Rep. 2014, 9, 2352-2356. [CrossRef]

10. He, C.; Deng, J.; Hu, X.; Zhou, S.; Yang, X. Vitamin A Inhibits the Action of LPS on Intestinal Epithelial Barrie Function and Tight Junction Proteins. Food Funct. 2019, 10, 1235-1242. [CrossRef] [PubMed]

11. Wang, W.; Zhao, J.; Gui, W.; Sun, D.; Dai, H.; Xiao, L.; Chu, H.; Du, F.; Zhu, Q.; Schnabl, B. Tauroursodeoxycholic acid inhibits intestinal inflammation and barrier disruption in NAFLD mice. Br. J. Pharmacol. 2017, 175, 469-484. [CrossRef]

12. Al-Sadi, R.M.; Ma, T.Y. IL-1beta causes an increase in intestinal epithelial tight junction permeability. J. Immunol. 2007, 178, 4641-4649. [CrossRef]

13. Tossetta, G.; Paolinelli, F.; Avellini, C.; Salvolini, E.; Ciarmela, P.; Lorenzi, T.; Emanuelli, M.; Toti, P.; Giuliante, R.; Gesuita, R. IL-1 $\beta$ and TGF- $\beta$ weaken the placental barrier through destruction of tight junctions: An in vivo and in vitro study. Placenta 2014, 35, 509-516. [CrossRef]

14. Endo, H.; Niioka, M.; Kobayashi, N.; Tanaka, M.; Watanabe, T. Butyrate-producing probiotics reduce nonalcoholic fatty liver disease progression in rats: New insight into the probiotics for the gut-liver axis. PLoS ONE 2013, 8, e63388. [CrossRef]

15. Jing, Y.; Yu, Y.; Bai, F.; Wang, L.; Yang, D.; Zhang, C.; Qin, C.; Yang, M.; Zhang, D.; Zhu, Y.; et al. Effect of fecal microbiota transplantation on neurological restoration in a spinal cord injury mouse model: Involvement of brain-gut axis. Microbiome 2021, 9, 59. [CrossRef] [PubMed]

16. da Silva, T.F.; Casarotti, S.N.; de Oliveira, G.L.V.; Penna, A.L.B. The impact of probiotics, prebiotics, and synbiotics on the biochemical, clinical, and immunological markers, as well as on the gut microbiota of obese hosts. Crit. Rev. Food. Sci. Nutr. 2021, 61, 337-355. [CrossRef] 
17. Sato, Y.; Kuroki, Y.; Oka, K.; Takahashi, M.; Rao, S.; Sukegawa, S.; Fujimura, T. Effects of Dietary Supplementation With Enterococcus faecium and Clostridium butyricum, Either Alone or in Combination, on Growth and Fecal Microbiota Composition of Post-weaning Pigs at a Commercial Farm. Front. Vet. Sci. 2019, 6, 26. [CrossRef] [PubMed]

18. Molnár, A.; Such, N.; Farkas, V.; Pál, L.; Menyhárt, L.; Wágner, L.; Husvéth, F.; Dublecz, K. Effects of Wheat Bran and Clostridium butyricum Supplementation on Cecal Microbiota, Short-Chain Fatty Acid Concentration, $\mathrm{pH}$ and Histomorphometry in Broiler Chickens. Animals 2020, 10, 2230. [CrossRef] [PubMed]

19. Vinolo, M.A.R.; Rodrigues, H.G.; Nachbar, R.T.; Curi, R. Modulation of inflammatory and immune responses by short-chain fatty acids. Diet Immun. Inflamm. 2013, 17, 435-458.

20. Hu, S.; Wang, J.; Xu, Y.; Yang, H.; Wang, J.; Xue, C.; Yan, X.; Su, L. Anti-inflammation effects of fucosylated chondroitin sulphate from Acaudina molpadioides by altering gut microbiota in obese mice. Food Funct. 2019, 10, 1736-1746. [CrossRef] [PubMed]

21. Abdel-Latif, M.A.; Abd El-Hack, M.E.; Swelum, A.A.; Saadeldin, I.M.; Elbestawy, A.R.; Shewita, R.S.; Ba-Awadh, H.A.; Alowaimer, A.N.; Abd El-Hamid, H.S. Single and Combined Effects of Clostridium butyricum and Saccharomyces cerevisiae on Growth Indices, Intestinal Health, and Immunity of Broilers. Animals 2018, 8, 184. [CrossRef] [PubMed]

22. Liu, L.; Zeng, D.; Yang, M.; Wen, B.; Lai, J.; Zhou, Y.; Sun, H.; Xiong, L.; Wang, J.; Lin, Y.; et al. Probiotic Clostridium butyricum Improves the Growth Performance, Immune Function, and Gut Microbiota of Weaning Rex Rabbits. Probiotics Antimicrob. Proteins 2018, 11, 1278-1292. [CrossRef] [PubMed]

23. Ding, J.; Dai, R.; Yang, L.; He, C.; Xu, K.; Liu, S.; Zhao, W.; Xiao, L.; Luo, L.; Zhang, Y.; et al. Inheritance and Establishment of Gut Microbiota in Chickens. Front. Microbiol. 2017, 8, 1967. [CrossRef]

24. Li, J.; Yuan, J.; Miao, Z.; Song, Z.; Yang, Y.; Tian, W.; Guo, Y. Effect of Dietary Nutrient Density on Small Intestinal Phosphate Transport and Bone Mineralization of Broilers during the Growing Period. PLoS ONE 2016, 11, e0153859. [CrossRef] [PubMed]

25. Li, Z.; Wang, W.; Liu, D.; Guo, Y. Effects of Lactobacillus acidophilus on the growth performance and intestinal health of broilers challenged with Clostridium perfringens. J. Anim. Sci. Biotechnol. 2018, 9, 25. [CrossRef]

26. Zhang, L.; Zhang, L.; Zhan, X.A.; Zeng, X.; Zhou, L.; Cao, G.; Chen, A.G.; Yang, C. Effects of dietary supplementation of probiotic, Clostridium butyricum, on growth performance, immune response, intestinal barrier function, and digestive enzyme activity in broiler chickens challenged with Escherichia coli K88. J. Anim. Sci. Biotechnol. 2016, 7, 1-9. [CrossRef] [PubMed]

27. Cao, G.; Tao, F.; Hu, Y.; Li, Z.; Zhang, Y.; Deng, B.; Zhan, X. Positive effects of a Clostridium butyricum-based compound probiotic on growth performance, immune responses, intestinal morphology, hypothalamic neurotransmitters, and colonic microbiota in weaned piglets. Food Funct. 2019, 10, 2926-2934. [CrossRef]

28. Wilson, F.D.; Cummings, T.S.; Barbosa, T.M.; Williams, C.J.; Gerard, P.D.; Peebles, E.D. Comparison of two methods for determination of intestinal villus to crypt ratios and documentation of early age-associated ratio changes in broiler chickens. Poult. Sci. 2018, 97, 1757-1761. [CrossRef]

29. Nunes, C.; Freitas, V.D.; Almeida, L.M.; Laranjinha, J. Red wine extract preserves tight junctions in intestinal epithelial cells under inflammatory conditions: Implications for intestinal inflammation. Food Funct. 2019, 10, 1364-1374. [CrossRef]

30. Jun, G.U.; Liu, J.B.; Huo, W.Z. BBI blocks LPS-mediated inhibitory effect on tight junction protein of intestinal epithelial cells. Chin. J. Infect. Control. 2018, 17, 185-190. (In Chinese)

31. Lucke, A.; Böhm, J.; Zebeli, Q.; Metzler-Zebeli, B.U. Dietary Deoxynivalenol Contamination and Oral Lipopolysaccharide Challenge Alters the Cecal Microbiota of Broiler Chickens. Front. Microbiol. 2018, 9, 804. [CrossRef]

32. Berni, C.; Canani, M.; Costanzo, L.; Leone, M.; Pedata, R. Potential beneficial effects of butyrate in intestinal and extraintestinal diseases. World J. Gastroenterol. 2011, 17, 1519-1528.

33. Zhang, W.H.; Jiang, Y.; Zhu, Q.F.; Gao, F.; Dai, S.F.; Chen, J.; Zhou, G.H. Sodium butyrate maintains growth performance by regulating the immune response in broiler chickens. Br. Poult. Sci. 2011, 52, 292-301. [CrossRef] [PubMed]

34. Zhou, Z.Y.; Packialakshmi, B.; Makkar, S.K.; Dridi, S.; Rath, N.C. Effect of butyrate on immune response of a chicken macrophage cell line. Vet. Immunol. Immunopathol. 2014, 162, 24-32. [CrossRef] [PubMed]

35. Herron, L.R.; Pridans, C.; Turnbull, M.L.; Smith, N.; Lillico, S.; Sherman, A.; Gilhooley, H.J.; Wear, M.; Kurian, D.; Papadakos, G. A chicken bioreactor for efficient production of functional cytokines. BMC Biotechnol. 2018, 18, 82. [CrossRef] [PubMed]

36. Fata, G.L.; Weber, P.; Mohajeri, M.H. Probiotics and the Gut Immune System: Indirect Regulation. Probiotics Antimicrob. Proteins 2018, 10, 11-21. [CrossRef]

37. Bauer, E.; Williams, B.A.; Smidt, H.; Verstegen, M.W.; Mosenthin, R. Influence of the gastrointestinal microbiota on development of the immune system in young animals. Curr. Issues Intest. Microbiol. 2006, 7, 35-51.

38. Miao, R.X.; Zhu, X.X.; Wan, C.M.; Wang, Z.L.; Wen, Y.; Li, Y.Y. Effect of Clostridium butyricum supplementation on the development of intestinal flora and the immune system of neonatal mice. Exp. Ther. Med. 2018, 15, 1081-1086.

39. Cresci, G.A.; Thangaraju, M.; Mellinger, J.D.; Liu, K.; Ganapathy, V. Colonic Gene Expression in Conventional and Germ-Free Mice with a Focus on the Butyrate Receptor GPR109A and the Butyrate Transporter SLC5A8. J. Gastrointest. Surg. 2010, 14, 449-461. [CrossRef]

40. Hasnain, S.Z.; Tauro, S.; Das, I.; Tong, H.; Chen, A.C.H.; Jeffery, P.L.; Mcdonald, V.; Florin, T.H.; Mcguckin, M.A. IL-10 Promotes Production of Intestinal Mucus by Suppressing Protein Misfolding and Endoplasmic Reticulum Stress in Goblet Cells. Gastroenterology 2013, 144, 357-368. [CrossRef] [PubMed] 
41. Kimiaeitalab, M.V.; Goudarzi, S.M.; Jiménez-Moreno, E.; Cámara, L.; Mateos, G.G. A comparative study on the effects of dietary sunflower hulls on growth performance and digestive tract traits of broilers and pullets fed a pullet diet from 0 to 21 days of age. Anim. Feed Sci. Technol. 2018, 236, 57-67. [CrossRef]

42. Wang, W.; Li, Z.; Lv, Z.; Zhang, B.; Lv, H.; Guo, Y. Effects of Kluyveromyces marxianus supplementation on immune responses, intestinal structure and microbiota in broiler chickens. PLoS ONE 2017, 12, e0180884. [CrossRef]

43. Wang, J.; Tang, H.; Zhang, C.; Zhao, Y.; Derrien, M.; Rocher, E.; van-Hylckama Vlieg, J.E.; Strissel, K.; Zhao, L.; Obin, M.; et al. Modulation of gut microbiota during probiotic-mediated attenuation of metabolic syndrome in high fat diet-fed mice. ISME J. 2015, 9, 1-15. [CrossRef] [PubMed]

44. Yang, Y.; Iji, P.A.; Choct, M. Dietary modulation of gut microflora in broiler chickens: A review of the role of six kinds of alternatives to in-feed antibiotics. World's Poult. Sci. J. 2009, 65, 97-114. [CrossRef]

45. Wang, Y.; Sun, J.; Zhong, H.; Li, N.; Xu, H.; Zhu, Q.; Liu, Y. Effect of probiotics on the meat flavour and gut microbiota of chicken. Sci. Rep. 2017, 7, 6400. [CrossRef]

46. Tanca, A.; Manghina, V.; Fraumene, C.; Palomba, A.; Abbondio, M.; Deligios, M.; Silverman, M.; Uzzau, S. Metaproteogenomics Reveals Taxonomic and Functional Changes between Cecal and Fecal Microbiota in Mouse. Front. Microbiol. 2017, 8, 391. [CrossRef] [PubMed]

47. Wang, S.; Chen, L.; He, M.; Shen, J.; Li, G.; Tao, Z.; Wu, R.; Lu, L. Different rearing conditions alter gut microbiota composition and host physiology in Shaoxing ducks. Sci. Rep. 2018, 8, 7387. [CrossRef]

48. Zhang, M.; Sun, K.; Wu, Y.; Yang, Y.; Tso, P.; Wu, Z. Interactions between Intestinal Microbiota and Host Immune Response in Inflammatory Bowel Disease. Front. Immunol. 2017, 8, 942. [CrossRef]

49. Liu, P.; Zhao, J.; Wang, W.; Guo, P.; Lu, W.; Wang, C.; Liu, L.; Johnston, L.J.; Zhao, Y.; Wu, X.; et al. Dietary Corn Bran Altered the Diversity of Microbial Communities and Cytokine Production in Weaned Pigs. Front. Microbiol. 2018, 9, 2090. [CrossRef]

50. Pandit, R.J.; Hinsu, A.T.; Patel, N.V.; Koringa, P.G.; Jakhesara, S.J.; Thakkar, J.R.; Shah, T.M.; Limon, G.; Psifidi, A.; Guitian, J.; et al. Microbial diversity and community composition of caecal microbiota in commercial and indigenous Indian chickens determined using 16s rDNA amplicon sequencing. Microbiome 2018, 6, 115. [CrossRef] 